\title{
Modeling and analysis of loaded multilayered magneto-electro-elastic structures composite materials: Applications
}

\author{
M. Ajdour ${ }^{1 *}$, A. Bakkali², L. Azrar ${ }^{3 ; 4}$ A. El Omri ${ }^{1}$ \\ ${ }^{1}$ Laboratory of Mechanics and Materials, Faculty of Sciences and Technology of Tangier, AbdelMalek Essaadi University; \\ Tangier; Morocco \\ ${ }^{2}$ Department of Physics, Faculty of Sciences of Tetouan, AbdelMalek Essaadi University; Tetouan. Morocco \\ ${ }^{3}$ LaMIPI, Higher School of Technical Education (ENSET) of Rabat, Mohammed V University in Rabat, Morocco \\ ${ }^{4}$ Department of Mechanical Engineering, Faculty of Engineering, King Abdulaziz University, Jeddah, Saudi Arabia \\ *corresponding author, majdour@hotmail.fr
}

\begin{abstract}
The aim of this study is to develop a two-scale tool allowing the detailed analysis of the behavior of fiber-reinforced magneto-electro-elastic composite plates. The work is divided into two major sections. The first one deals with the homogenization of the properties of each layer based on the Mori-Tanaka mean field approach where all needed effective coefficients of each layer are determined. In the second one and in order to perform the analysis of the behavior of the obtained magneto-electro-elastic multilayered plate, the Stroh formalism is used. It allows to predict the effective behavior of such plates and the spatial distribution of the local fields along the layers.
\end{abstract}

\section{Introduction}

The behavior of active materials often exhibits multiphysical coupling effect. Moreover, the use of composite materials is increasingly used to combine the different advantages of each material.

Magneto-electro-elastic composites represent a new class of materials with several potential applications in modern nanoscience and nanotechnology. The interaction between electric polarization and magnetization offers new possibilities for functional materials such sensors and actuators.

In addition to being a multiphysical material and in order to analyze materials with magneto-electric coupling, it is important to be able to determine the distribution of the physical fields within these heterogeneous structures [1].

Many analytical and mathematical models are developed to predict new heterogeneous magneto-electro-elastic composite materials. $\mathrm{Li}$ [2] studied the average magnetoelectro-elastic field in a multi-inclusion or inhomogeneities embedded in an infinite matrix. Feng, et al. [3] investigated the effective properties of composite consisting of piezomagnetic inhomogeneities embedded in a non-piezomagnetic matrix by using a unified energy method and the Mori-Tanaka and Dilute approaches. Zhang and Soh [4] extended the micromechanical Self Consistent, Mori-Tanaka and Dilute to study the coupled magneto-electro-elastic composite materials. The effective properties of multiphase and coated magneto-electro-elastic heterogeneous materials have been investigated by Bakkali et al [5] based on various micromechanical models. Some approaches have been proposed to deal with fully coupled magneto-electro-elastic laminates. Several explicit expressions have been found by Kim [6] to calculate the magnetic, electric, elastic, piezoelectric, magneto-elastic and magneto-electric effective properties. On the other hand, similar results have been obtained in [7, 8]. More recently, L.M. Sixto-Camacho et al [9] use the asymptotic homogenization to derive the local problems and the corresponding homogenized coefficients of periodic thermo-magneto-electro-elastic heterogeneous media. The theory is applied to obtain analytical expressions for all effective properties of an important class of periodic multilaminated composites.

The Mori Tanaka model presented in this paper is used to predict the effective magneto-electro-elastic coefficients. This models permit to take into account the effect of phase number and concentrations, shape inclusions, as well as its polling orientation. Results for a two-phase composite material (Piezo-electric/Piezo-magnetic) with fibrous microstructure are presented.

The macroscale equilibrium equations are solved analytically using the Stroh formalism [10-11] associated with the propagation matrix. It should be noted that a same analysis has been proposed by [15] to deal with piezoelectric fiber actuators. However, in this multiscale analysis the behavior of each layer was obtained by periodic homogenization, which needs a more inextricable numerical procedure.

This formalism will provide solutions for multifunctional multilayered plate, to predict the mechanical, electrical and magnetic behaviors near or across the interface of material layers.

The coupled multiscale analysis procedure is illustrated through two model problems. The first model problem presents the behavior of a sandwich plate made of three heterogonous magneto-electro-elastic layers under a surface mechanical load. The second problem describes the evolution of some physical properties of graded material 
under a surface mechanical load composed by six heterogeneous magneto-electro-elastic layers.

These numerical results should be of interest to the design of magnet-electro-elastic composite laminates.

\section{Constitutive laws and equilibrium equations of magneto-electro-elastic material}

The constitutive equations for the magneto-electro-elastic medium relating stress $\sigma_{i j}$, electric displacement $D_{i}$ and magnetic induction $B_{i}$ to strain $\varepsilon_{k l}$, electric field $E_{k}$ and magnetic field $\mathrm{H}_{1}$, exhibiting linear coupling between magnetic, electric and elastic field can be written as:

$$
\begin{aligned}
& \sigma_{i j}=C_{i j k l} \varepsilon_{k l}-e_{k i j} E_{k}-h_{l i j} H_{l} \\
& D_{i}=e_{i k l} \varepsilon_{k l}+\kappa_{i l} E_{l}+\alpha_{i l} H_{l} \\
& B_{i}=h_{i k l} \varepsilon_{k l}+\alpha_{i l} E_{l}+\mu_{i l} H_{l}
\end{aligned}
$$

where the elastic strain $\varepsilon_{k l}$, electric fields $E_{l}$ and the magnetic fields $H_{l}$ are independent variables related to stresses $\sigma_{i j}$, electric displacements $D_{i}$ and magnetic inductions $B_{i}$. The tensors $C_{i j k l}, e_{l i j}, \alpha_{i l}, h_{i l}, \kappa_{i l}$ and $\mu_{i l}$ are the elastic, piezo-electric, piezo-magnetic, magnetoelectric, dielectric and magnetic permeability constants respectively. The following gradient expressions are used:

$$
\varepsilon_{k l}=\frac{1}{2}\left(u_{k, l}+u_{l, k}\right), E_{l}=-\phi_{, l}^{e} \text { and } H_{l}=-\phi_{, l}^{m}
$$

where $u_{k}, \varphi^{e}$ and $\varphi^{m}$ are the elastic displacements, electric and magnetic potentials respectively. The notation $a_{, l}$ means $\frac{\partial a}{\partial x_{l}}$.

In order to make easy the manipulation of these equations, particular notations will be used. These notations are identical to those using the conventional subscripts except that the lower case subscripts assume the range of $1-3$, while capital subscripts take the range of 1-5, and repeated capital subscripts are summed over 1-5.

With these notations, the magneto-electro-elastic constant can be represented as follows [5]:

$$
E_{i J M n}= \begin{cases}C_{i j m n} & (J, M=1,2,3) \\ e_{n i j} & (J=1,2,3 ; M=4) \\ h_{n i j} & (J=1,2,3 ; M=5) \\ e_{i m n} & (J=4 ; M=1,2,3) \\ h_{i m n} & (J=5 ; M=1,2,3) \\ -\kappa_{i n} & (J=4 ; M=4) \\ -\alpha_{i n} & (J=4 ; M=5 \text { or } J=5 ; M=4) \\ -\mu_{i n} & (J=5 ; M=5)\end{cases}
$$

The generalized strain field denoted by $Z_{M n}$ can be expressed as:

$$
Z_{M n}= \begin{cases}\varepsilon_{m n} & (M=1,2,3) \\ -E_{n} & (M=4) \\ -H_{n} & (M=5)\end{cases}
$$

Similarly, the generalized stress field $\Sigma_{\mathrm{i} J}$ is given by:

$$
\Sigma_{i J}= \begin{cases}\sigma_{i j} & (J=1,2,3) \\ D_{i} & (J=4) \\ B_{i} & (J=5)\end{cases}
$$

The equations of equilibrium, in the absence of body force and free charge and current, can be written as:

$$
\begin{aligned}
\sigma_{i j, j} & =0 \\
D_{j, j} & =0 \\
B_{j, j} & =0
\end{aligned}
$$

In what follows we will study the response of a multilayer subjected to uniaxial loading while considering each layer as a magneto-electro-elastic material composed of either a piezo-magnetic matrix with different volume fraction of piezo-electric inclusion, or a piezo-electric matrix with different volume fraction of inclusion piezo-magnetic.

\section{Micromechanics modelling}

In this section, the effective properties of two kinds of magneto-electro-elastic composites are computed based on the Mori-Tanaka micromechanical mean field approach. The first one is constituted of a piezo-magnetic matrix $\left(\mathrm{CoFe}_{2} \mathrm{O}_{4}\right)$ reinforced by aligned fibrous piezo-electric inclusions $\left(\mathrm{BaTiO}_{3}\right)$ and the second one is constituted of a piezo-electric matrix $\left(\mathrm{BaTiO}_{3}\right)$ reinforced by aligned fibrous piezo-magnetic inclusions $\left(\mathrm{CoFe}_{2} \mathrm{O}_{4}\right)$. The micromechanics modeling is divided on two steps: The localization step which relate the local fields with the global ones and the homogenization step which is based on average techniques. A representative volume element $\mathrm{V}$ of the composite is considered. The Macroscopic fields are related to the local ones by the mean operator:

$$
\begin{aligned}
& Z_{I J}^{0}=\frac{1}{V} \int_{V} Z_{I J}(r) d V \\
& \sum_{i J}^{0}=\frac{1}{V} \int_{V} \sum_{i J}(r) d V
\end{aligned}
$$

For an N-phase composite, the macroscopic fields are reformulated as:

$$
\begin{aligned}
& Z_{I J}^{0}=\sum_{i=1}^{N} f_{i} Z_{I J}^{i} \\
& \sum_{I J}^{0}=\sum_{i=1}^{N} f_{i} \sum_{I J}^{i}
\end{aligned}
$$

where ' $\mathrm{i}$ ' points to the $\mathrm{i}^{\text {th }}$ phase and $f_{i}$ is the associated volume fraction. $Z^{i}$ and $\sum^{i}$ represent the local uniform fields.

Moreover, the overall constitutive equations that represent the effective behavior of the composite and each of its constituent (phase $\mathrm{p}$ ) are given respectively by: 


$$
\begin{aligned}
\sum_{i J}^{0} & =E_{i J K l}^{e f f} Z_{K L}^{0} \\
\sum_{i J}^{p} & =E_{i J K l}^{p} Z_{K l}^{p}
\end{aligned}
$$

In order to make the transition scale between the local uniform fields (phases) and the macroscopic fields (composite), the localization tensors are introduced. One can write the localization equations as follow [5].

$$
\begin{aligned}
& Z_{K l}^{p}=A_{K I M n}^{p} Z_{M n}^{0} \\
& \sum_{i J}^{p}=B_{i J n M}^{p} \sum_{n M}^{0}
\end{aligned}
$$

Based on averaging techniques (Eqs. 7 and 8) and using the localization equations (Eq. 10), the expression of the effective properties is obtained:

$$
E_{k L S v}^{e f f}=\sum_{p=1}^{N} f_{p} E_{k L L j}^{p} A_{l j S v}^{p}
$$

For the case of the magneto-electro-elastic composite considered in this article, the expression of the effective properties is given by:

$$
E_{i J K l}^{e f f}=E_{i J K l}^{M}+f^{I}\left(E_{i J M n}^{I}-E_{i J M n}^{M}\right) A_{M n K l}
$$

where $E_{i I M n}^{I}$ and $f^{I}$ represent the properties of the inclusions and its associated volume fraction and $E_{i J M n}^{M}$ represents the properties of the matrix. The localization tensor is a function of the phases' properties as well as of the shape of the inclusions. The localization tensor could be estimated based on different micromechanical models. In this paper the Mori-Tanaka model, known to be accurate and of its ease in implementation, is considered. Its expression is given by [5].

$$
A_{M n K L}=\left(I_{K I M n}+\frac{f^{M}}{V^{I}} T_{i J K L}^{I I} \Delta E_{i J M n}^{I}\right)^{-1}
$$

in which, $\Delta E_{i M n n}^{I}=E_{i J M n}^{I}-E_{i M n n}^{m}$ and $T^{I I}$ is the magnetoelectro-elastic interaction tensor that is function of the properties of the matrix and the shape of the inclusion. Details about the computation of the interaction tensor are given in [5].

Some Numerical results are presented bellow for the considered magneto-electro-elastic composites [5]. The used properties are listed in table 1 . The electro-magnetic moduli are presented in figure 1. The evolution of these coefficients versus the volume fraction of the piezo-electric inclusions is shown.

A numerical data of the effective coefficients for different volume fractions of inclusions is also presented in table 2 and table 3.

Table 1: Piezo-electric and piezo-magnetic material properties $\left(\mathrm{C}_{\mathrm{ij}}\right.$ in $\mathrm{GPa}$; $\kappa_{\mathrm{ij}}$ in $10^{-9} \mathrm{C}^{2} / \mathrm{Nm}^{2} ; \mu_{\mathrm{ij}}$ in $10^{-6}$ $\mathrm{Ns}^{2} / \mathrm{C}^{2}$, $e_{\mathrm{ij}}$ in $\mathrm{C} / \mathrm{m}^{2}$ and $\mathrm{h}_{\mathrm{ij}}$ in $\left.\mathrm{N} / \mathrm{Am}\right)$.

\begin{tabular}{ccc}
\hline $\begin{array}{c}\text { Material } \\
\text { property }\end{array}$ & $\mathbf{B a T i O}_{3}$ & $\mathbf{C o F e}_{2} \mathbf{O}_{\mathbf{4}}$ \\
\hline $\mathrm{C}_{11}$ & 166 & 286 \\
$\mathrm{C}_{13}$ & 77 & 173 \\
$\mathrm{C}_{12}$ & 78 & 170 \\
$\mathrm{C}_{22}$ & 162 & 269 \\
$\mathrm{C}_{44}$ & 43 & 45 \\
$\mathrm{e}_{16}$ & 11.5 & 0 \\
$\mathrm{e}_{21}$ & -4.4 & 0 \\
$\mathrm{e}_{22}$ & 18.6 & 0 \\
\hline
\end{tabular}

\begin{tabular}{ccc}
$\kappa_{11}$ & 11.2 & 0.08 \\
$\kappa_{22}$ & 12.6 & 0.093 \\
$\mathrm{~h}_{16}$ & 0 & 550 \\
$\mathrm{~h}_{21}$ & 0 & 580.3 \\
$\mathrm{~h}_{22}$ & 0 & 699.7 \\
$\mu_{11}$ & 5 & -590 \\
$\mu_{22}$ & 10 & 157 \\
\hline
\end{tabular}
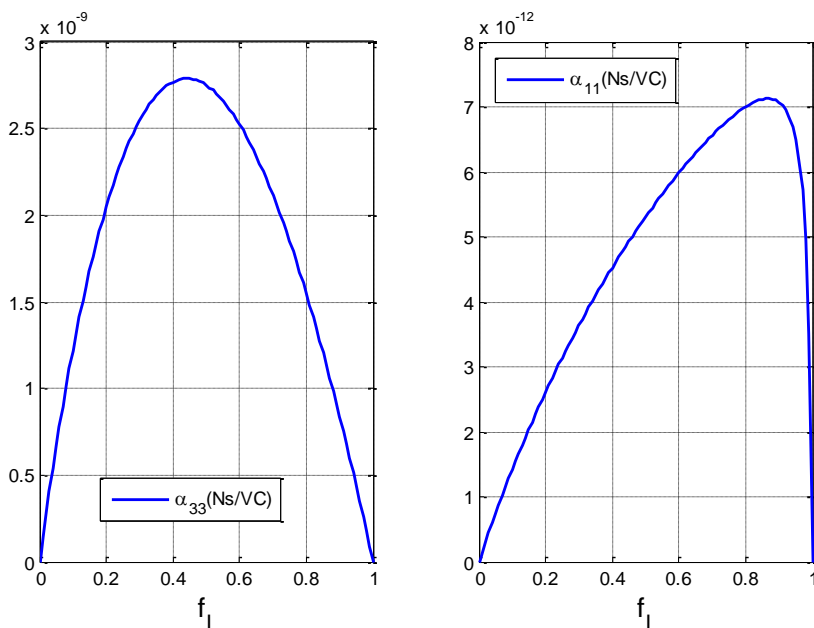

Figure 1: The effective magneto-electric moduli $\alpha_{11}$ et $\alpha_{33}$ presented for a fibrous magneto-electro-elastic composite constituted of piezo-electric inclusions embedded in a piezomagnetic matrix versus the volume fraction of the piezoelectric inclusions.

\section{Stroh formalism solution for the macroscopic fields}

In order to analyze and design materials and devices with magneto-electric coupling, it is important to determine the distribution of the physical fields within these heterogeneous structures.

We use the Stroh formalism, described by Ting [11], to obtain a general solution of Eq (6). The state variables satisfy the case of simply supported boundary condition.

Solutions of extended displacement vector and traction vector are respectively assumed to be as follow:

$$
\begin{gathered}
u=\left[\begin{array}{l}
u_{1} \\
u_{2} \\
u_{3} \\
\varphi \\
\psi
\end{array}\right]=e^{s z}\left[\begin{array}{l}
a_{1} \cos p x \sin q y \\
a_{2} \sin p x \cos q y \\
a_{3} \sin p x \sin q y \\
a_{4} \sin p x \cos q y \\
a_{5} \sin p x \cos q y
\end{array}\right] \\
t=\left[\begin{array}{l}
\sigma_{13} \\
\sigma_{23} \\
\sigma_{33} \\
D_{3} \\
B_{3}
\end{array}\right]=e^{s z}\left[\begin{array}{l}
b_{1} \cos p x \sin q y \\
b_{2} \sin p x \cos q y \\
b_{3} \sin p x \sin q y \\
b_{4} \sin p x \cos q y \\
b_{5} \sin p x \cos q y
\end{array}\right]
\end{gathered}
$$

where $\mathrm{p}=\mathrm{n} \pi / \mathrm{L}_{\mathrm{x}}, \mathrm{q}=\mathrm{m} \pi / \mathrm{L}_{\mathrm{y}} ; \mathrm{n}$ and $\mathrm{m}$ are two positive integers. 
Based on the Stroh formalism [10-11], the vector $b=\left[b_{1}, b_{2}, b_{3}, b_{4}, b_{5}\right]$ is related to the vector $a=\left[a_{1}, a_{2}, a_{3}, a_{4}, a_{5}\right]$ by:

$$
b=\left(-R^{T}+s T\right) a=-\frac{1}{s}(Q+s R) a
$$

where:

$$
R=\left[\begin{array}{ccccc}
0 & 0 & p C_{13} & 0 & 0 \\
0 & 0 & q C_{23} & 0 & 0 \\
-p C_{55} & -q C_{44} & 0 & -q e_{34} & -q h_{34} \\
0 & 0 & q e_{23} & 0 & 0 \\
0 & 0 & q h_{23} & 0 & 0
\end{array}\right]
$$

$$
T=\left[\begin{array}{ccccc}
C_{55} & 0 & 0 & 0 & 0 \\
& C_{44} & 0 & e_{34} & h_{34} \\
& & C_{33} & 0 & 0 \\
& & & -\kappa_{33} & -\alpha_{33} \\
& & & & -\mu_{33}
\end{array}\right]
$$

$$
Q=\left[\begin{array}{ccccc}
-p^{2} C_{11}-q^{2} C_{66} & -\left(C_{12}+C_{66}\right) p q & 0 & -\left(e_{16}+e_{21}\right) p q & -p q\left(h_{16}+h_{21}\right) \\
& -q^{2} C_{66}-p^{2} C_{22} & 0 & -p^{2} e_{16}-q^{2} e_{22} & -p^{2} h_{16}-q^{2} h_{22} \\
& & -p^{2} C_{55}-q^{2} C_{44} & 0 & 0 \\
& & & \kappa_{11} p^{2}+\kappa_{22} q^{2} & \alpha_{11} p^{2}+\alpha_{22} q^{2} \\
& & & & \mu_{11} p^{2}+\mu_{22} q^{2}
\end{array}\right]
$$

Stresses should satisfy the equations of equilibrium, which in terms of the vector $\mathbf{a}$, yields the following eigenequation:

$$
\left[Q+s\left(R+R^{T}\right)+s^{2} T\right] a=0
$$

The linear eigensystem to be solved is then:

$$
N\left\lfloor\begin{array}{l}
a \\
b
\end{array}\right\rfloor=s\left\lfloor\begin{array}{l}
a \\
b
\end{array}\right\rfloor
$$

where

$$
N=\left[\begin{array}{cc}
-T^{-1} R^{T} & T^{-1} \\
-Q+R T^{-1} R^{T} & -R T^{-1}
\end{array}\right]
$$

In order to obtain the extended displacement and traction vectors at any depth, say $z_{k} \leq z \leq z_{k+1}$ in layer $\mathrm{k}$, we propagate the solution from the bottom of the surface to the z-level, i.e.,

$\left\lfloor\begin{array}{l}u \\ t\end{array}\right\rfloor_{H}=P_{k}\left(z-z_{k-1}\right) P_{k-1}\left(h_{k-1}\right) \ldots \ldots P_{2}\left(h_{2}\right) P_{1}\left(h_{1}\right)\left\lfloor\begin{array}{l}u \\ t\end{array}\right\rfloor_{0}$

where $h_{j}=z_{j+1}-z_{j}$ is the thickness of layer $\mathrm{j}$.

The propagating relation can be used repeatedly so that one can propagate the physical quantities from the bottom surface $\mathrm{z}=0$ to the top surface $\mathrm{z}=\mathrm{H}$ of the layered plate, then:

$$
\left\lfloor\begin{array}{l}
u \\
t
\end{array}\right\rfloor_{H}=P_{N}\left(h_{N}\right) P_{N-1}\left(h_{N-1}\right) \ldots \ldots P_{2}\left(h_{2}\right) P_{1}\left(h_{1}\right)\left\lfloor\left[\begin{array}{l}
u \\
t
\end{array}\right\rfloor_{0}\right.
$$

Various combinations of mechanical and electrical loads may be considered at the top $(\mathrm{z}=\mathrm{H})$ and at the bottom $(\mathrm{z}=0)$ of the plate.

The Eshelby-Stroh solution for the macroscale analysis of laminated piezo-electric composite structures has been used in earlier studies [12, 13, 14]

\section{Two-scale method results}

The multiscale framework is used to analyze two model problems. In the first problem, we consider a simply supported laminate consisting on sandwich multilayered composed by three magneto-electro-elastic layers of equal thickness $\mathrm{h}=0.1$ with Piezo-electric fiber volume fraction respectively of $0.1,0.5$ and 0.1 (figure 2). A z-direction traction with amplitude $\sigma_{0}=1 \mathrm{~N} / \mathrm{m}^{2}$ is applied on the top of the surface $\mathrm{z}=0.3 \mathrm{~m}$. Responses are calculated for fixed horizontal coordinates $(\mathrm{x}, \mathrm{y})=\left(0.75 \mathrm{~L}_{\mathrm{x}}, 0.25 \mathrm{~L}_{\mathrm{y}}\right)$.

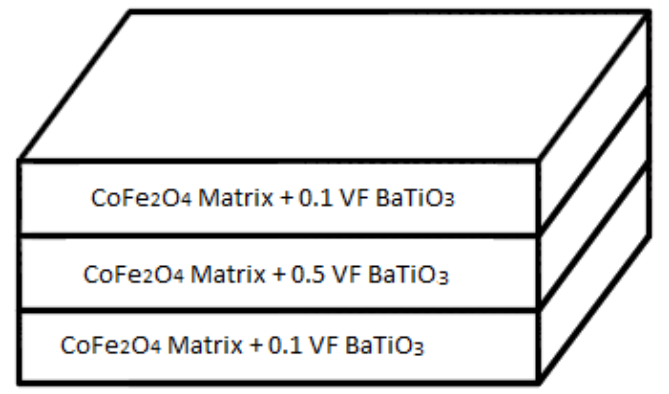

Figure 2: Sandwich multilayer made by piezo-magnetic matrix with Piezo-electric fiber volume fraction (VF) respectively of $0.1,0.5$ and 0.1

This case will be compared to the one where the matrix is made by a piezo-electric material and the inclusion made by a piezo-magnetic material (figure 3 ).

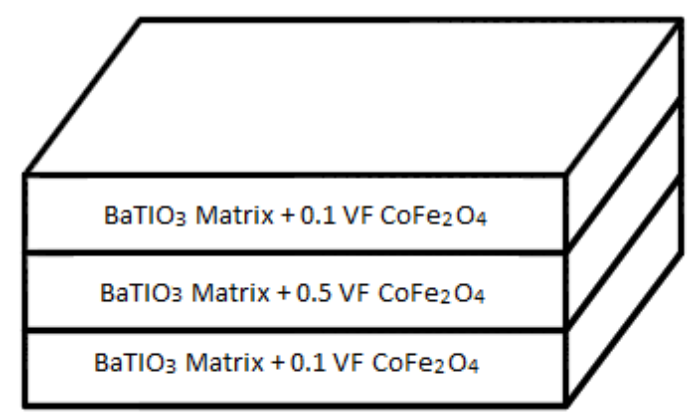

Figure 3: Sandwich multilayer made by piezo-electric matrix with Piezo-electric fiber volume fraction (VF) respectively of $0.1,0.5$ and 0.1 
Table 2: Effective properties of fibrous Magneto-electro-elastic composites constituted of a piezomagnetic $\left(\mathrm{CoFe}_{2} \mathrm{O}_{4}\right)$ matrix reinforced by piezo-electric (BaTiO3) inclusions $\left(\mathrm{C}_{\mathrm{ij}}\right.$ in $\mathrm{GPa} ; \kappa_{\mathrm{ij}}$ in $10^{-9} \mathrm{C}^{2} / \mathrm{Nm}^{2} ; \mu_{\mathrm{ij}}$ in $\mathrm{Ns}^{2} / \mathrm{C}^{2}, \mathrm{e}_{\mathrm{ij}}$ in $\mathrm{C} / \mathrm{m}^{2} ; \mathrm{h}_{\mathrm{ij}}$ in $\mathrm{N} / \mathrm{Am}$ and $\alpha \mathrm{ij}$ in $\left.10^{-9} \mathrm{Ns} / \mathrm{VC}\right)$.

\begin{tabular}{|c|c|c|c|c|}
\hline $\begin{array}{l}\text { Material } \\
\text { property }\end{array}$ & $\mathrm{VF}=0.1$ & $V F=0.15$ & $\mathrm{VF}=0.3$ & $\mathrm{VF}=0.5$ \\
\hline $\mathrm{C}_{11}$ & 268.29 & 260.12 & 238.01 & 213.02 \\
\hline $\mathrm{C}_{22}$ & 254.72 & 247.82 & 228.81 & 206.63 \\
\hline $\mathrm{C}_{44}$ & 46.108 & 46.524 & 47.811 & 49.592 \\
\hline $\mathrm{C}_{55}$ & 55.145 & 54.482 & 52.548 & 50.093 \\
\hline $\mathrm{C}_{13}$ & 158 & 151.1 & 132.91 & 112.84 \\
\hline $\mathrm{C}_{12}$ & 156.06 & 149.68 & 132.48 & 113.29 \\
\hline $\mathrm{e}_{21}$ & -0.6659 & -0.9712 & -1.79316 & -2.7108 \\
\hline $\mathrm{e}_{22}$ & 1.66569 & 2.52231 & 5.17274 & 8.8598 \\
\hline $\mathrm{e}_{16}$ & 0.01645 & 0.02624 & 0.06451 & 0.1525 \\
\hline $\mathrm{h}_{21}$ & 492.395 & 452.108 & 343.657 & 222.61 \\
\hline $\mathrm{h}_{22}$ & 604.279 & 559.692 & 436.501 & 292.31 \\
\hline $\mathrm{h}_{16}$ & 449.222 & 405.509 & 294.832 & 182.131 \\
\hline $\mathrm{K}_{11}$ & 0.09753 & 0.1078 & 0.14731 & 0.2358 \\
\hline $\mathrm{K}_{22}$ & 1.3519 & 1.9803 & 3.8621 & 6.3646 \\
\hline$\mu_{11}$ & -0.000481 & -0.000434 & -0.00031 & -0.00019 \\
\hline$\mu_{22}$ & 0.00014 & 0.000135 & 0.000113 & $8.386 e-5$ \\
\hline$\alpha_{11}$ & $1.41 \mathrm{e}-3$ & $2.0319 \mathrm{e}-3$ & $3.6321 \mathrm{e}-3$ & $5.306 \mathrm{e}-3$ \\
\hline$\alpha_{22}$ & 1.22 & 1.6749 & 2.5466 & 2.7499 \\
\hline
\end{tabular}

Table 3: Effective properties of fibrous magneto-electroelastic composites constituted of a piezo-electric (BaTiO $\left.\mathbf{B}_{3}\right)$ matrix reinforced by piezo-magnetic $\left(\mathrm{CoFe}_{2} \mathrm{O}_{4}\right)$ inclusions $\left(\mathrm{C}_{\mathrm{ij}}\right.$ in $\mathrm{GPa} ; \kappa_{\mathrm{ij}}$ in $10^{-9} \mathrm{C}^{2} / \mathrm{Nm}^{2} ; \mu_{\mathrm{ij}}$ in $\mathrm{Ns}^{2} / \mathrm{C}^{2}, \mathrm{e}_{\mathrm{ij}}$ in $\mathrm{C} / \mathrm{m}^{2} ; \mathrm{h}_{\mathrm{ij}}$ in $\mathrm{N} / \mathrm{Am}$ and $\alpha \mathrm{ij}$ in $10^{-9} \mathrm{Ns} / \mathrm{VC}$ ).

\begin{tabular}{crrrc}
\hline $\begin{array}{l}\text { Material } \\
\text { property }\end{array}$ & \multicolumn{1}{l}{$\mathbf{V F}=0.1$} & $\mathbf{V F}=0.15$ & $\mathbf{V F}=0.3$ & $\mathbf{V F}=0.5$ \\
\hline $\mathrm{C}_{11}$ & 173.85 & 178 & 191.48 & 212.27 \\
$\mathrm{C}_{22}$ & 169.86 & 173.94 & 186.89 & 206.13 \\
$\mathrm{C}_{44}$ & 43.841 & 44.183 & 44.942 & 45.429 \\
$\mathrm{C}_{55}$ & 45.54 & 46.072 & 47.715 & 50.027 \\
$\mathrm{C}_{13}$ & 82.772 & 85.86 & 96.046 & 112.22 \\
$\mathrm{C}_{12}$ & 83.802 & 86.886 & 96.964 & 112.71 \\
$\mathrm{e}_{21}$ & -4.12249 & -3.97501 & -3.493045 & -2.73999 \\
$\mathrm{e}_{22}$ & 16.6015 & 15.6097 & 12.6681 & 8.83996 \\
$\mathrm{e}_{16}$ & 9.83339 & 9.05335 & 6.892 & 4.3814 \\
$\mathrm{~h}_{21}$ & 36.5939 & 56.0411 & 119.6 & 218.912 \\
$\mathrm{~h}_{22}$ & 51.7065 & 78.5398 & 163.485 & 289.158 \\
$\mathrm{~h}_{16}$ & -0.91335 & -1.47788 & -3.7768 & -9.32543 \\
$\mathrm{~K}_{11}$ & 9.1895 & 8.3138 & 6.0869 & 3.8041 \\
$\mathrm{~K}_{22}$ & 11.356 & 10.734 & 8.8647 & 6.3685 \\
$\mu_{11}$ & $6.13 \mathrm{e}-06$ & $6.8 \mathrm{e}-06$ & $9.391 \mathrm{e}-06$ & $1.535 \mathrm{e}-05$ \\
$\mu_{22}$ & $2.48 \mathrm{e}-05$ & $3.221 \mathrm{e}-05$ & $5.439 \mathrm{e}-05$ & $8.388 \mathrm{e}-05$ \\
$\alpha_{11}$ & -0.034183 & -0.04446 & -0.055479 & -0.041491 \\
$\alpha_{22}$ & 0.87296 & 1.2626 & 2.2191 & 2.9012 \\
\hline & & & &
\end{tabular}

Figures 4 and 5 present the evolution of the electric and magnetic potential along the thickness direction of these different sandwich multilayers. It is obvious that the potential variations for the two cases are completely different.

Figure 4 shows that the two composites behave in a different manner except at the intermediate layer where the volume fraction of the both inclusions is the same.

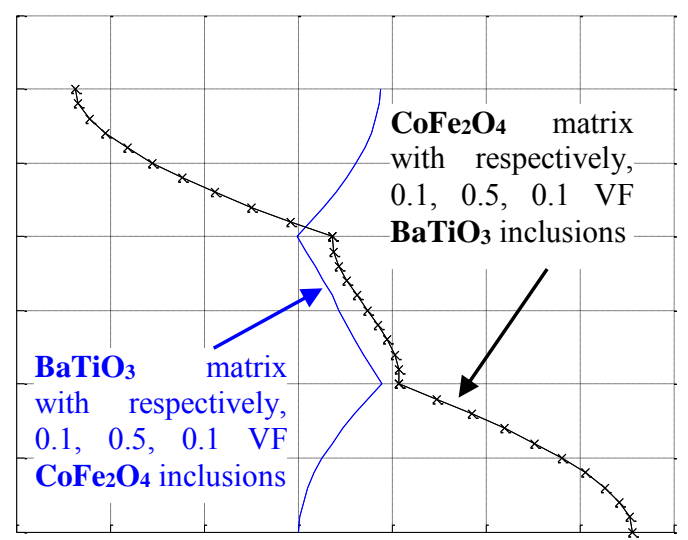

Figure 4 : Variation of the electric potential along the thickness direction in the sandwich plate caused by a surface load on the top surface

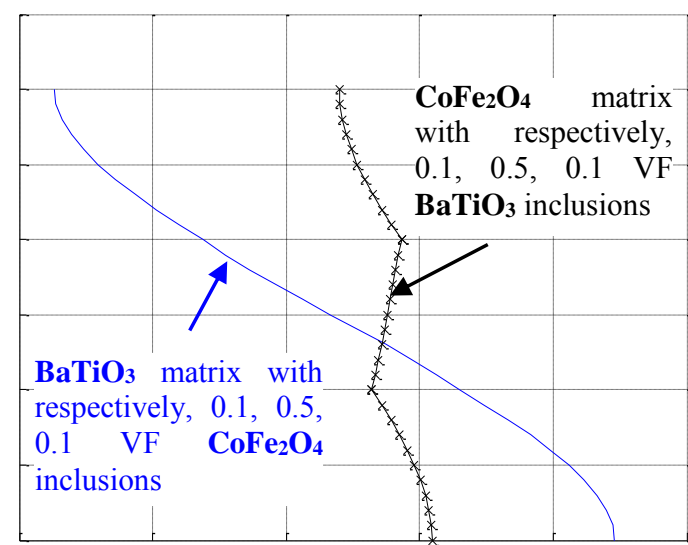

Figure 5: Variation of the magnetic potential along the thickness direction in the sandwich plate caused by a surface load on the top surface

The second model concerns the graded material shown in figure 6 .

In this example, we consider a simply supported laminate consisting on a graded material composed by six magneto-electro-elastic layers of equal thickness $\mathrm{h}=0.05 \mathrm{~m}$. The first three layers are made by piezo-magnetic $\left(\mathrm{CoFe}_{2} \mathrm{O}_{4}\right)$ matrix and piezo-electric $\left(\mathrm{BaTiO}_{3}\right)$ inclusions with volume fraction ranging from 0.15 to 0.5 . The last three layers are each made by a piezo-electric (BaTiO3) matrix and piezo-magnetic $\left(\mathrm{CoFe}_{2} \mathrm{O}_{4}\right)$ inclusions with volume fraction varying from 0.5 to 0.15 (figure 6 ). A z-direction 
traction with amplitude $\sigma_{0}=1 \mathrm{~N} / \mathrm{m}^{2}$ is applied on the top of the surface $\mathrm{z}=0.3 \mathrm{~m}$. Responses are calculated for fixed horizontal coordinates $(\mathrm{x}, \mathrm{y})=\left(0.75 \mathrm{~L}_{\mathrm{x}}, 0.25 \mathrm{~L}_{\mathrm{y}}\right)$.

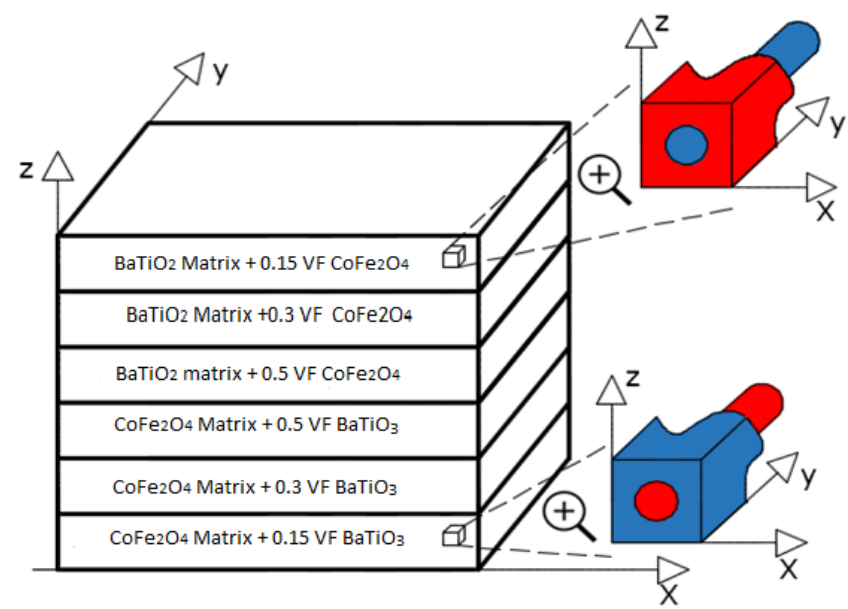

Figure 6: Graded material

The behavior of this multilayer is compared with that of a composite consisting of layers made by piezo-magnetic $\left(\mathrm{CoFe}_{2} \mathrm{O}_{4}\right)$ matrix with a 0.5 volume fraction of piezoelectric $\left(\mathbf{B a T i O}_{3}\right)$ inclusion and to that of a composite of layers made by piezo-electric matrix with 0.5 volume fraction of piezo-magnetic inclusion.

Figures 7 and 8 show the evolution of the electric and magnetic potential for these different multilayers. We observe that the evolution of the electrical potential of the three first layers of the graded material varies in the same direction as in the case of composite made by a piezomagnetic matrix with 0.5 volume fraction of piezo-electric inclusion.

However, for the last three layers the electric potential varies in the same direction as the case of composite made by piezo-electric matrix with 0.5 volume fraction piezomagnetic inclusion.

$\mathrm{CoFe}_{2} \mathrm{O}_{4}$ matrix with 0.5

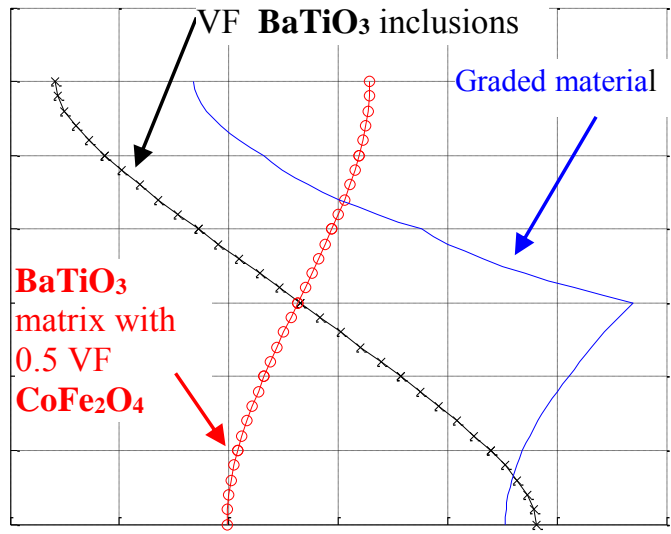

Figure 7 : Variation of the electric potential along the thickness direction in the graded material caused by a surface load on the top surface

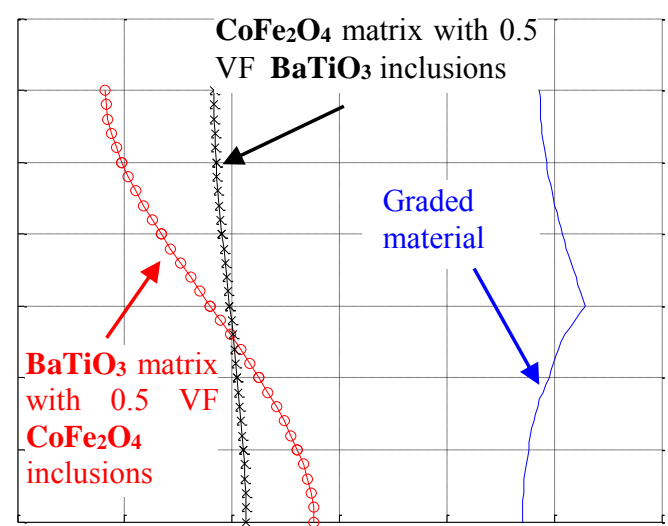

Figure 8 : Variation of the magnetic potential along the thickness direction in the graded material caused by a surface load on the top surface

\section{Conclusion}

In this paper, the micro-macro problem to obtain the homogenized effective coefficients of magneto-electroelastic heterogeneous media was derived based on the Mori Tanaka method. This homogenization model is used to obtain the effective elastic, piezo-electric, piezo-magnetic, dielectric, magnetic and magneto-electric coefficients. Stroh Formalism is devoted to predict macroscopic fields in a multilayered plate.

This multiscale framework is used to analyze two problems, a sandwich multilayered plates and a graded material. This allowed us to predict the behavior of these multilayered rectangular plates under surface loads with defined inclusion direction.

Apart from extending the multiscale method proposed by [15] to taking into account the magnetic effect, the present method is based on a more simple procedure (Mori-Tanaka) to describe the behavior of each layer.

A genetic algorithm can be developed to optimize the best distribution of volume fraction of inclusion fiber for some defined physical constraints.

\section{References}

[1] R. Corcolle, L. Daniel, F. Bouillaut, Generic formalism for homogenization of coupled behavior: application to magneto-electro-elastic behavior. Physical Review B, 78: 214110, 2008.

[2] J.Y. Li, Magnetoelectroelastic multi-inclusion and inhomogeneity problems and their applications in composite materials. Int. J. of Engineering Science, 38: 1993-2011, 2000.

[3] X. Feng, D. Fang, K. Hwang, Closed-form solution for piezomagnetic inhomogeneities embedded in a nonpiezomagnetic matrix. European Journal of Mechanics A/Solids, 23: 1007-1019, 2004. 
[4] Z.K. Zhang, A.K. Soh, Micromechanics predictions of the effective moduli of magnetoelectroelastic composite materials, European. J. of Mechanics A/Solids, 24: 1054-1067, 2005.

[5] A. Bakkali, L. Azrar, A. Aljinaidi, Effective properties of heterogeneous magnetoelectroelastic materials with multiCoated inclusions. Key Engineering Materials, 550: 25-32, 2013.

[6] J.Y. Kim, Micromechanical analysis of effective properties of magneto-electro-thermo-elastic multilayer composites. International Journal of Engineering Science, 49: 1001-1018, 2011.

[7] J. Bravo-Castillero, R. Rodríguez-Ramos, H. Mechkour, J.A. Otero, F.J. Sabina, Homogenization of magneto-electro-elastic multilaminated materials, The Quarterly Journal of Mechanics and Applied Mathematics, 61: 311-332, 2008.

[8] K.S. Challagulla, A.V. Georgiades, Micromechanical analysis of magneto-electro-thermo-elastic composite materials with applications to multilayered structures, International Journal of Engineering Science, 4: 85-104, 2011.

[9] L.M. Sixto-Camacho, R. Bravo-Castillero, R. Brenner, R. Guinovart-Díaz, H. Mechkour, R. Rodríguez-Raos, F. J. Sabina, Asymptotic homogenization of periodic thermosmagneto-electro-elastic heterogeneous media, International Journal of Computers and Matrhematics with applications, 66: 2056-2074, 2013.

[10] A.N. Stroh. Dislocation and cracks in anisotropic elasticity, Philos. Mag, 625-646, 1958.

[11] T.C.T. Ting. Anisotropic Elasticity: Theory and Applications, Oxford University Press, Oxford U.K, 1996.

[12] SS. Vel, R.C. Mewer, R.C. Batra. Analytical solution for the cylindrical bending vibration of piezoelectric composite plates, International Journal of Solids and Structures, 41 :1625-1643, 2004.

[13] E. Pan, Exact Solution for Simply Supported and Multilayered Magneto-Electro-Elastic Plates, Journal of applied Mechanics, 68: 608-618, 2011.

[14] M. Ajdour, L. Azrar, Analytical Solutions of Loaded Multifunctional Multilayered Plates, Advanced Materials Research, 682: 127-134, 2013.

[15] A.C. Cook, SS. Vel, Multiscale analysis of laminated plates with integrated piezoelectric fiber composite actuators, Composite Structures, 94: 322 - 336, 2012. 